\title{
A standardized extract from Paeonia lactiflora and Astragalus membranaceus attenuates liver fibrosis induced by porcine serum in rats
}

\author{
WU-YI SUN, LING WANG, HAO LIU, XIANG LI and WEI WEI \\ Institute of Clinical Pharmacology, Anhui Medical University, Key Laboratory of Anti-inflammatory and \\ Immunophamacology of Education Ministry, Key Laboratory of Research and Development of Chinese \\ Medicine in Anhui Province, Engineering Technology Research Center of Anti-Inflammatory \\ and Immunodrugs in Anhui Province, Hefei 230032, Anhui Province, P.R. China
}

Received September 27, 2011; Accepted November 1, 2011

DOI: $10.3892 / \mathrm{ijmm} .2011 .844$

\begin{abstract}
Paeonia lactiflora and Astragalus membranaceus are two popular traditional Chinese medicines, commonly used in Chinese herb prescription to treat liver disease. The extract prepared from the roots of Paeonia lactiflora and Astragalus membranaceus (PAE) demonstrated better hepatoprotective activity than the herbs used individually as shown in our previous studies. This study was carried out to investigate the effects of PAE on liver fibrosis induced by porcine serum (PS) in rats and to explore its possible mechanisms. Liver fibrosis was induced in male Wistar rats by injection with PS intraperitoneally. The rats were randomly divided into a normal control group, a liver fibrosis model group and a PAE $\left(40,80,160 \mathrm{mg} \cdot \mathrm{kg}^{-1}\right)$ treated group. After a 16-week treatment, PAE-treated rats showed significantly reduced liver damage and symptoms of liver fibrosis upon pathological examination. Administration of PAE significantly decreased serum HA, PC III levels, and content of hydroxyproline in the liver tissue of fibrotic rats. It also restored the decrease in SOD and GSH-Px activities and inhibited the formation of lipid peroxidative products during PS treatment. In vitro, PAE also significantly decreased $\left[{ }^{3} \mathrm{H}\right]$-thymidine incorporation in hepatic stellate cells (HSCs) stimulated with platelet-derived growth factor-B subunit homodimer (PDGF-BB). Moreover, PAE significantly decreased the expression of PDGF receptor beta (PDGFR- $\beta$ ) and p-ERK1/2, p-p38, p-JNK. The results showed that PAE displays antifibrotic effects in rats induced by PS, the mechanism by which might be associated with its ability to scavenge free radicals, decreasing the expression of
\end{abstract}

Correspondence to: Professor Wei Wei, Institute of Clinical Pharmacology, Anhui Medical University, Hefei 230032, Anhui Province, P.R. China

E-mail: sunwuyi51@yahoo.com.cn; wwei@ahmu.edu.cn

Key words: Paeonia lactiflora, Astragalus membranaceus, liver fibrosis, hepatic stellate cell
PDGFR- $\beta$, inhibition of HSC proliferation and MAPK activation. These findings indicate that PAE is a potential agent for the prevention of liver fibrosis.

\section{Introduction}

Liver fibrosis is a major feature of many chronic liver injuries and is one of the main components of cirrhosis. It is characterized by the accumulation of collagen and extracellular matrix (ECM) proteins in the space of Disse. Activation of hepatic stellate cells (HSCs) and the initiation of unbalanced synthesis of collagen are the main components in the pathogenesis that leads to fibrosis $(1,2)$. Advanced liver fibrosis leads eventually to cirrhosis and liver failure, for which no effective medical treatments are available and is associated with high morbidity and mortality. Thus, it is essential to develop therapeutic strategies to counteract liver fibrosis (3).

Oxidative stress has recently been recognized as a fundamental factor in the pathological changes observed in various liver diseases $(4,5)$. Free radicals, formed as a result of oxidative stress, cause extensive damage to DNA, proteins, lipids and carbohydrates, leading to various acute and chronic liver injuries (6). Several lines of evidence have suggested the important role of oxidative stress in the etiopathogenesis of liver fibrosis (7). Furthermore, oxidative stress aggravates liver fibrosis via HSCs activation, and lipid peroxidation stimulates transcription of the collagen gene (8).

The standardized extract from Paeonia lactiflora and Astragalus membranaceus (PAE) is made up of paeony glycoside and astragalosides extracted from Paeonia lactiflora pall and Astragalus membranaceus (Fisch.) Bge., with a standard ratio of $4: 1$. Studies in our laboratory have shown that paeony glycoside, extracted from Paeonia lactiflora pall root retarded the progression of experimental immunological hepatic fibrosis through inhibition of collagen synthesis and decreasing oxidative stress, but had modest effect on HSCs proliferation (9). Astragalosides effectively retarded the progression of experimental hepatic fibrosis through inhibition of HSCs proliferation and collagen production (10). In traditional oriental medicine, it is conventional to combine different herbs in order to achieve 
a variety of treatment purposes simultaneously, or to enhance a single effect without causing severe side effects (11). PAE was developed to maximize the hepato-protective effect of the two herbs, and our previous animal study confirmed that PAE exhibited the most significant hepato-protective activity compared to exclusively using Paeonia lactiflora or Astragalus membranaceus (12). Our previous studies have shown that PAE has protective effects on chemical liver injury and BCG/ LPS-induced liver injury in mice through its ability to scavenge free radicals and inhibit pro-inflammatory mediator and cytokines production. In addition, PAE also enhances the levels of potential antioxidants such as glutathione peroxidase (GSH-Px), superoxide dismutase (SOD) $(13,14)$. These studies have resulted in considerable interest in PAE as a therapeutic agent in chronic liver disease.

At present, no clinically effective therapy for liver fibrosis is available. We hypothesize that PAE could be a potential therapeutic candidate for fibrotic liver diseases. Therefore, this study was designed to further evaluate the effects of PAE on porcine serum (PS) induced liver fibrosis in rats and to propose its possible mechanism of action.

\section{Materials and methods}

Preparation of PAE. Radix Paeonia lactiflora and Radix Astragali were purchased from Hefei Heyitang Pharmacy, China. The production areas of Paeonia lactiflora pall and Astragalus membranaceus (Fisch.) Bge. were Anhui Province and Shanxi Province (China) respectively. Voucher specimens (Pan 2009002 and Pan 2009013) were identified by Professor Lumin Pan in Department of Materia Madica, School of Pharmacy, Anhui College of Traditional Chinese Medicine and deposited in the Chinese Materia Madica Specimen Center of Anhui College of Traditional Chinese Medicine. The processes of extracting and preparing PAE (composed of paeony glycoside and astragalosides with a standard ratio of 4:1) from the two herbs were as described previously (13). Before administration to rats, PAE was dissolved in $0.5 \%$ sodium carboxymethylcellulose solutions.

Animals and treatment. Male Wistar rats, weighing 120-140 g, were provided by the Shanghai BK Experimental Animal Center (Grade II, Certificate no. D-65). All animals were housed in conventional cages under control conditions of temperature $\left(23 \pm 3^{\circ} \mathrm{C}\right)$ and relative humidity $(50 \pm 20 \%)$, with light illumination for $12 \mathrm{~h} /$ day. All experimental protocols described in this study were approved by the Ethics Review Committee for Animal Experimentation of Institute of Clinical Pharmacology, Anhui Medical University (ER2009031201). After 1 week of acclimatization, the rats were randomly divided into five experimental groups. The animals were allowed access to food and tap water ad libitum throughout the acclimatization and experimental periods.

Hepatic fibrosis was induced in rats by repeated intraperitoneal injection of $0.5 \mathrm{ml}$ of PS (Gibco-BRL, Life Technologies, Rockville, MD) twice a week for up to 16 weeks (15). The rats were randomly divided into five groups. Group 1 was normal control, Group 2 was PS model, Groups 3, 4 and 5 were PS and PAE (40, 80 and $160 \mathrm{mg} / \mathrm{kg}$ ) treated rats. Groups $2-5$ received intraperitoneal injection of $0.5 \mathrm{ml}$ of PS for 16 weeks. Group 2 was fed the basal diet throughout the experiment and was designed as a model group. Groups 3-5 were treated with PAE (40, 80 and $160 \mathrm{mg} / \mathrm{kg}$ ) during the entire experimental period. The control group, which received repeated intraperitoneal injection of $0.5 \mathrm{ml}$ saline, was administered with the same volume of vehicle.

At $24 \mathrm{~h}$ after final injection of PS, all rats were anaesthetized with diethyl ether, samples of blood were drawn from the abdominal aorta and collected in polyethylene tubes. The serum samples obtained by centrifugation $\left(3,000 \mathrm{x} \mathrm{g}, 4^{\circ} \mathrm{C}\right)$ for $10 \mathrm{~min}$ and were kept frozen at $-80^{\circ} \mathrm{C}$ until assayed as described below. After the animals were sacrificed, the liver was promptly removed. A portion of the liver was fixed for histopathology, and the remaining tissues were stored at $-80^{\circ} \mathrm{C}$ until required.

Assessment of serum enzyme activities. The serum activities of alanine aminotransferase (ALT) and aspartate aminotransferase (AST) were estimated using commercially available kits (Jiancheng Biologic Co., Nanjing, China).

Hepatic hydroxyproline content. Liver tissue (100 mg) was prepared for hydroxyproline (Hyp) determination according to a modified method by Mitchell and Taylor (16). The Hyp content of the liver as an indirect measure of tissue collagen content was expressed as $\mathrm{mg} / \mathrm{g}$ wet tissue.

Serum levels of fibrotic markers. Serum levels of hyaluronic acid (HA) and procollagen type III (PCIII) were determined by radioimmunoassay (RIA) using commercially available kits (Beijing North Institute of Biological Technology, Beijing, China) according to the manufacturer's instructions.

Histopathological examination. Immediately after sacrifice, the liver tissue was removed and a portion of the tissue was instantly fixed in $10 \%$ phosphate-buffered formalin, processed by routine histology procedures, embedded in paraffin, cut in $5 \mu \mathrm{m}$ pieces and mounted on the slide. The samples were stained with hematoxylin and eosin (H\&E), Masson for histopathological examination. Two pathologists who had no knowledge of their sources examined the stained slides independently. Histological grade of hepatic fibrosis was determined by a semi-quantitative method according to the following scoring system (17): 0 , no fibrosis, normal liver and absence of fibrosis; I, fibrosis present (collagen fiber present that extends from portal triad or central vein to peripheral region); II, mild fibrosis (mild collagen fiber present with extension without compartment formation); III, moderate fibrosis (moderate collagen fiber present with some pseudo lobe formation); IV, severe fibrosis (severe collagen fiber present with thickening of the partial compartments and frequent pseudo lobe formation). Each sample was observed at x100 magnification. The degree of fibrosis was expressed as the mean of 10 different fields in each slide.

Estimation of antioxidant enzyme and lipid peroxidation. Liver tissues were washed with normal saline to remove any red blood and clots, and then were homogenized on ice with Tris- $\mathrm{HCl}$ (5 mM containing, $2 \mathrm{mM}$ EDTA, $\mathrm{pH}$ 7.4). Homogenates were centrifuged at $1,000 \mathrm{x} \mathrm{g}$ for $15 \mathrm{~min}$ at $4^{\circ} \mathrm{C}$. Aliquots of the supernatants were analyzed for antioxidant enzymes and lipid 


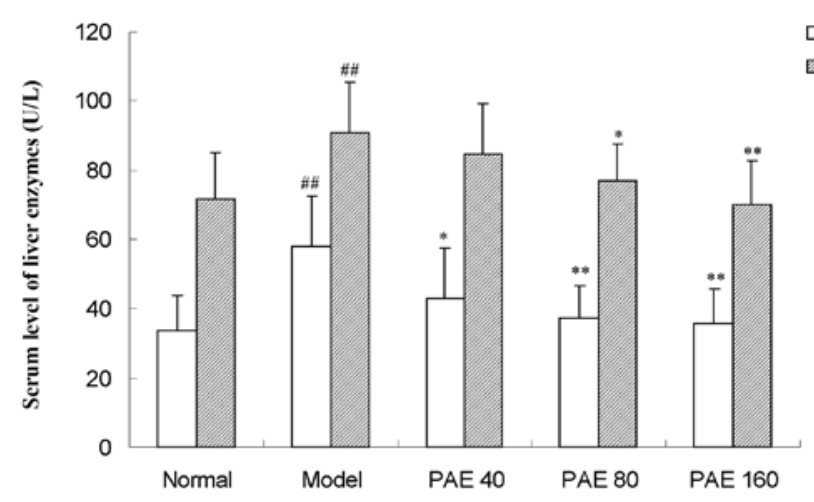

Figure 1. Effect of PAE on serum ALT and AST activities of liver fiborsis rats $(\mathrm{n}=8$; mean $\pm \mathrm{SD}) .{ }^{\# \#} \mathrm{P}<0.01$ compared with normal control group; ${ }^{*} \mathrm{P}<0.05$, ${ }^{* *} \mathrm{P}<0.01$ compared with model group.

peroxidation. For the determination of antioxidant enzymes, we measured the activities of SOD and GSH-Px based on their ability to inhibit the oxidation of oxyamine by the xanthinexanthine oxidase system. The results are expressed as the units for SOD or GSH-Px/milligram of liver tissue. Lipid peroxidation in the liver was determined by measuring the level of malondialdehyde (MDA), an end product of lipid peroxidation, using a thiobarbiturate method. The level of hepatic MDA was expressed as $\mu \mathrm{mol} / \mathrm{g}$ protein. The operations were performed according to the manufacturer's instructions (Jiancheng Biologic Co., Nanjing, China).

Culture of HSCs and proliferation assay. In vitro studies were performed on an immortalized rat HSC cell line (HSC-T6). The HSC-T6 line was cultured at $37^{\circ} \mathrm{C}$ in an atmosphere of $5 \% \mathrm{CO}_{2}$ in Dulbecco's modified minimal essential medium (Gibco-BRL) containing 10\% fetal calf serum (FCS), $2 \mathrm{mmol} / \mathrm{l}$ L-glutamine, and 5,000 IU/ml penicillin $/ 5,000 \mathrm{~g} / \mathrm{ml}$ streptomycin for 1 to 2 days before starting experiments. HSC-T6 had a pseudomyofibroblastic phenotype, characteristic cell shape, and expression of $\alpha$-SMA (18).

To evaluate HSCs proliferation, we measured the intracellular uptake of $\left[{ }^{3} \mathrm{H}\right]$-thymidine. HSC-T6 were plated at a density of $1.0 \times 10^{5}$ cells $/ \mathrm{ml}$ in 96 -well culture plates. The confluent cells were growth-arrested in thymidine-free medium (DMEM containing $0.5 \%$ bovine serum albumin) for $24 \mathrm{~h}$. Subsequently, cells were incubated with $50 \mathrm{ng} / \mathrm{ml}$ PDGF-BB and either vehicle or a given concentration of PAE (20, 40, 80, $160 \mathrm{mg} / \mathrm{l})$. Meanwhile, $1 \mu \mathrm{Ci} / \mathrm{ml}$ of $\left[{ }^{3} \mathrm{H}\right]$-thymidine (China Institute of Atomic Energy, Beijing, China) was added, followed by incubation at $37^{\circ} \mathrm{C}$ in $5 \% \mathrm{CO}_{2}$ for $24 \mathrm{~h}$. Washed with PBS, the HSCs were digested with trypsin and absorbed on glass fiber filter paper. The cells were then washed once with $10 \%$ trichloroacetic acid and three times with saline. The uptake of $\left[{ }^{3} \mathrm{H}\right]$-thymidine was measured using a liquid scintillation counter. HSCs cultured in PDGF-BB free DMEM mixed with $1 \mu \mathrm{Ci} / \mathrm{ml}$ of $\left[{ }^{3} \mathrm{H}\right]$-thymidine for $24 \mathrm{~h}$ were used as a control. Six cultures in each group were examined.

Western blotting. Approximately $50 \mathrm{mg}$ of the liver sample was homogenized in RIPA lysis buffer ( $0.1 \%$ SDS, $0.5 \%$ deoxycholate, $1 \%$ Nonidet P-40, $100 \mathrm{mM} \mathrm{NaCl}, 10 \mathrm{mM}$ Tris-HCl pH 7.4) containing protease inhibitors $(1 \mathrm{mM}$ phenylmethylsulphonyl fluoride, $10 \mu \mathrm{g} / \mathrm{ml}$ aprotinin and $10 \mu \mathrm{g} / \mathrm{ml}$ leupeptin). Protein concentration was measured by the Bradford assay. Liver protein extracts from rats of each experimental group as well as normal controls were separated by $10 \%$ sodium dodecyl sulfatepolyacrylamide gel electrophoresis (SDS-PAGE) and transferred to polyvinylidene difluoride membranes (Millipore, Bedford, MA, USA). The membranes were then blocked with $5 \%$ non-fat dry milk in $0.05 \%$ Tween-20-PBS for $2 \mathrm{~h}$ and incubated overnight at $4^{\circ} \mathrm{C}$ with anti-ERK1/2, phospho-ERK1/2, p38, phospho-p38, JNK and phospho-JNK (Cell Signaling Technology, Danvers, MA, USA), plateletderived growth factor receptor $\beta$ (PDGFR- $\beta$ ) (Santa Cruz Biotechnology, Inc., Santa Cruz, CA, USA) antibodies. After washing with TPBS, the membranes were incubated for $2 \mathrm{~h}$ at room temperature with secondary HRP conjugated antibody (Zhongshan Goldenbridge Biotechnology, Beijing, China), and visualized using ECL detection kit (Pierce Chemical, Rockford, IL, USA). Monoclonal anti- $\beta$-actin antibody (Santa Cruz Biotechnology, Inc.) was used to confirm equal loading of protein. The density of the specific bands was quantified with an Image-Pro Plus software (Media Cybernetics, Silver Springs, MD, USA).

Statistical analysis. Data were expressed as means \pm SD. Statistical analysis of the data for multiple comparisons was performed by one-way ANOVA followed by Dunnett's test. The frequency data were compared using Ridit procedure. $\mathrm{P}<0.05$ indicated a statistically significant difference.

\section{Results}

Effect of PAE on liver function. ALT and AST activities at the end of the experiment are shown in Fig. 1. In PS model group, the activities of ALT and AST were significantly increased compared with normal control group. PAE $(80,160 \mathrm{mg} / \mathrm{kg}$, intragastrically) decreased the elevated ALT and AST activities in serum.

Effect of PAE on hydroxyproline content in liver. Analysis of hepatic hydroxyproline content was carried out as an index of liver fibrosis. As shown in Table I, liver fibrosis induced by PS caused significant rise in hydroxyproline content, whereas PAE treatment significantly attenuated the increased level of the hydroxyproline.

Effect of PAE on serum HA and PCIII. Serum levels of HA and PCIII are surrogate markers of liver fibrogenesis. As shown in Table I, compared with normal rats, the model control rats exhibited higher levels of serum HA and PCIII. Administration with PAE $(80,160 \mathrm{mg} / \mathrm{kg})$ caused a significant decrease in serum levels of HA and PCIII, while PAE (40 mg/ $\mathrm{kg}$ ) did not have significant effect.

Histological results. In order to examine whether treatment of rats with PS alone for 16 weeks induces hepatic fibrosis and the protect effects of PAE, tissue sections from livers in each group were stained with H\&E and Masson staining. Representative photographs of the liver morphology are shown in Fig. 2. The control group showed normal lobular architecture with central veins and radiating hepatic cords (Fig. 2A and D). In contrast, 
Table I. Effect of PAE on serum HA, PCIII levels and hepatic hydroxyproline content in immunological hepatic fibrosis rats induced by porcine serum (mean $\pm \mathrm{SD} ; \mathrm{n}=8$ ).

\begin{tabular}{lcccc}
\hline Group & $\begin{array}{c}\text { Dose } \\
\left(\mathrm{mg} \cdot \mathrm{kg}^{-1}\right)\end{array}$ & $\begin{array}{c}\text { HA } \\
\left(\mu \mathrm{g} \cdot \mathrm{l}^{-1}\right)\end{array}$ & $\begin{array}{c}\text { PCIII } \\
\left(\mu \mathrm{g} \cdot \mathrm{l}^{-1}\right)\end{array}$ & $\begin{array}{c}\text { Hydroxyproline } \\
\left.\left(\mathrm{mg}^{-1} \mathrm{~g} \mathrm{pr}\right)^{-1}\right)\end{array}$ \\
\hline Normal & - & $190.38 \pm 33.20$ & $18.51 \pm 5.94$ & $0.35 \pm 0.11$ \\
Model & - & $461.53 \pm 69.12^{\mathrm{a}}$ & $37.76 \pm 8.42^{\mathrm{a}}$ & $0.98 \pm 0.29^{\mathrm{a}}$ \\
PAE & 40 & $394.30 \pm 64.66$ & $31.27 \pm 6.10$ & $0.72 \pm 0.13^{\mathrm{b}}$ \\
& 80 & $348.01 \pm 84.24^{\mathrm{b}}$ & $24.87 \pm 7.55^{\mathrm{c}}$ & $0.62 \pm 0.10^{\mathrm{c}}$ \\
& 160 & $265.28 \pm 67.24^{\mathrm{c}}$ & $25.69 \pm 8.31^{\mathrm{b}}$ & $0.60 \pm 0.11^{\mathrm{c}}$ \\
\hline
\end{tabular}

${ }^{\mathrm{a}} \mathrm{P}<0.01$ compared with normal group; ${ }^{\mathrm{b}} \mathrm{P}<0.05,{ }^{\mathrm{C}} \mathrm{P}<0.01$ compared with model group.
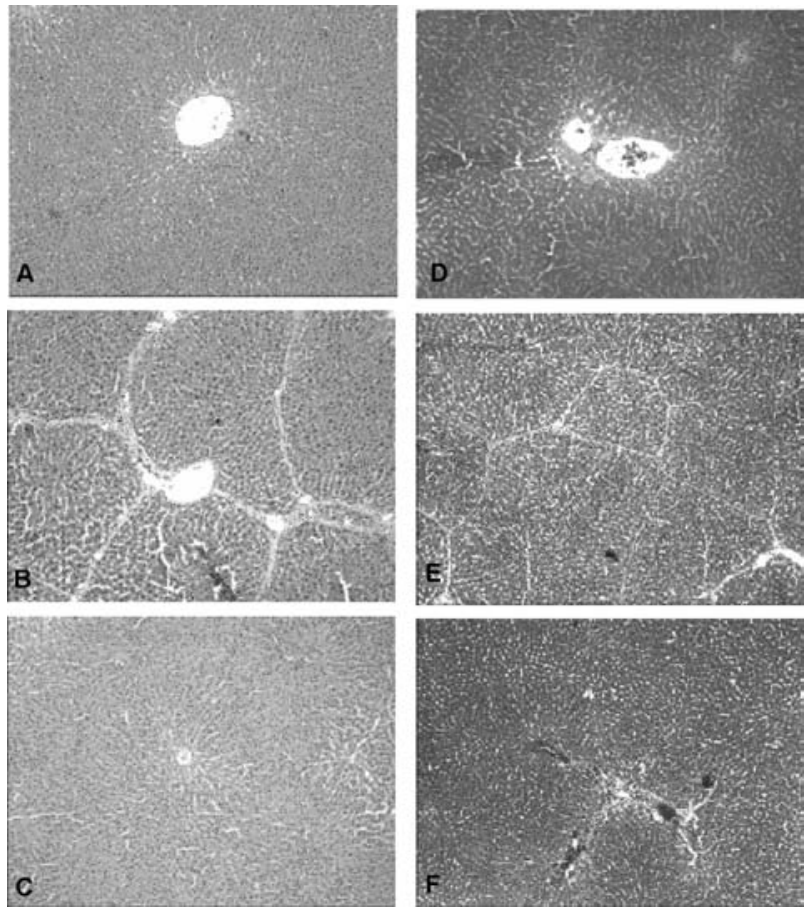

Figure 2. Effect of PAE on the histological morphology of fibrotic rat liver taken from the experimental groups. (A) Normal control. (B) PS model. (C) PAE $(160 \mathrm{mg} / \mathrm{kg}$ ) H\&E staining, original magnification, x100. (D) Normal control. (E) PS model. (F) PAE (160 mg/kg) Masson stain, original magnification, $x 100$.

livers in model group showed thick fibrotic septa connecting portal tracts, delimiting the classic liver lobule. The hepatic lobules were encysted and separated by collagen bundles. The normal structure of lobules was destroyed and even pseudolobule formation (Fig. 2B and E). No obvious hepatocellular necrosis was found. Treatment with PAE $(160 \mathrm{mg} / \mathrm{kg})$ resulted in apparent amelioration of the degree of liver fibrosis indicated by decreased collagen deposition with no obvious pseudolobuli formation (Fig. 2C and F). Statistical analysis also presented significant differences between PAE (80, $160 \mathrm{mg} / \mathrm{kg}$ ) and model group in histological scores, indicating that fibrogenesis in PAE treatment group was much less severe than that of model group (Table II).

Effect of PAE on hepatic lipid peroxidation and SOD, GSH-Px activity. Data on lipid peroxidation in terms of MDA forma-

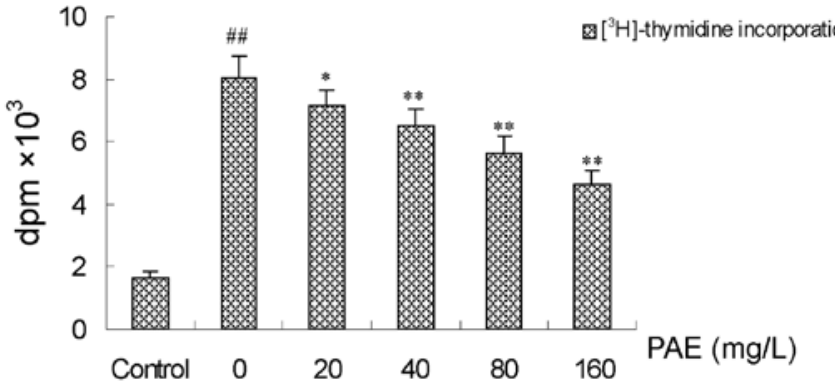

Figure 3. Effect of PAE on PDGF-BB stimulates HSC proliferation in vitro. $\left[{ }^{3} \mathrm{H}\right]$-thymidine incorporation (an index of proliferation) in HSCs incubated for $24 \mathrm{~h}$ with either DMEM alone (control), or $50 \mathrm{ng} / \mathrm{ml}$ PDGF-BB with a given concentration of PAE. ${ }^{\# /} \mathrm{P}<0.01$ compared with control group; ${ }^{*} \mathrm{P}<0.05,{ }^{* *} \mathrm{P}<0.01$ compared with cells treated with PDGF-BB alone. Values are mean \pm SD for six observations.

tion of whole liver homogenate of control and experimental animals are presented in Table III. A marked increase in MDA production was found in the liver of PS-treated animals relative to normal control group. PAE-treated rats at doses of 40 , 80 and $160 \mathrm{mg} / \mathrm{kg}$ significantly reduced MDA level.

At the end of the experiment the changes in SOD and GSH-Px activities were assayed in rat livers in order to evaluate endogenous antioxidant defences (Table III). A significant decrease in SOD and GSH-Px activity was observed in the liver of PS-treated rats relative to control rats administered with vehicle alone. Administration with PAE $(80,160 \mathrm{mg} / \mathrm{kg}$ ) significantly elevated the activities of both enzymes.

Effect of PAE on PDGF-BB stimulates proliferation in HSCs. PDGF-BB has a growth stimulatory effect on HSCs (19). The uptake of $\left[{ }^{3} \mathrm{H}\right]$-thymidine in HSCs with PDGF-BB was significantly increased compared with that in HSCs without PDGF-BB (Fig. 3). Under the condition where the growth of HSCs was stimulated by PDGF-BB, the effect of PAE was investigated. When various concentrations of $\operatorname{PAE}(20,40,80$, $160 \mathrm{mg} / \mathrm{l}$ ) were added to medium containing PDGF-BB, the uptake of $\left[{ }^{3} \mathrm{H}\right]$-thymidine was significantly inhibited.

Effect of PAE on PDGFR- $\beta$ expression in liver tissue. To elucidate the possible mechanisms in regard to how PAE attenuates liver fibrosis, the effects of this agent on the expression of 
Table II. Effects of PAE on the pathologic grading of immunological liver fibrosis rats induced by porcine serum (mean \pm SD; $\mathrm{n}=8$ )

\begin{tabular}{|c|c|c|c|c|c|c|c|}
\hline \multirow[b]{2}{*}{ Group } & \multirow{2}{*}{$\begin{array}{c}\text { Dose } \\
\left(\mathrm{mg} \cdot \mathrm{kg}^{-1}\right)\end{array}$} & \multicolumn{5}{|c|}{ Pathologic grading of hepatic fibrosis } & \multirow[b]{2}{*}{ P-value } \\
\hline & & 0 & I & II & III & IV & \\
\hline Normal & - & 8 & 0 & 0 & 0 & 0 & - \\
\hline Model & - & 0 & 0 & 1 & 4 & 3 & $0.000^{\mathrm{a}}$ \\
\hline \multirow[t]{3}{*}{ PAE } & 40 & 0 & 0 & 3 & 3 & 2 & 0.340 \\
\hline & 80 & 0 & 1 & 3 & 4 & 0 & $0.036^{\mathrm{b}}$ \\
\hline & 160 & 0 & 2 & 3 & 3 & 0 & $0.017^{\mathrm{b}}$ \\
\hline
\end{tabular}

Results are 10 fields of vision. ${ }^{\mathrm{a}} \mathrm{P}<0.01$ compared with normal control group; ${ }^{\text {b }}<0.05$ compared with model group.

Table III. Effect of PAE on MDA level, SOD and GSH-Px activities of liver homogenate in immunological hepatic fibrosis rats induced by porcine serum (mean $\pm \mathrm{SD} ; \mathrm{n}=8$ ).

\begin{tabular}{|c|c|c|c|c|}
\hline Group & $\begin{array}{c}\text { Dose } \\
\left(\mathrm{mg} \cdot \mathrm{kg}^{-1}\right)\end{array}$ & $\begin{array}{c}\text { MDA } \\
\left(\mu \mathrm{mol} \cdot \mathrm{g} \mathrm{pr}^{-1}\right)\end{array}$ & $\begin{array}{c}\text { SOD } \\
\left(\mathrm{U} \cdot \mathrm{mg} \mathrm{pr}^{-1}\right)\end{array}$ & $\begin{array}{c}\text { GSH-Px } \\
\left(\mathrm{U} \cdot \mathrm{mg} \mathrm{pr} \mathrm{pr}^{-1}\right)\end{array}$ \\
\hline Normal & - & $2.50 \pm 0.38$ & $134.83 \pm 14.30$ & $219.33 \pm 38.18$ \\
\hline Model & - & $4.96 \pm 0.81^{\mathrm{a}}$ & $90.38 \pm 14.19^{\mathrm{a}}$ & $93.75 \pm 25.84^{\mathrm{a}}$ \\
\hline \multirow[t]{3}{*}{ PAE } & 40 & $3.74 \pm 1.02^{\mathrm{b}}$ & $105.13 \pm 17.81$ & $120.10 \pm 22.99^{b}$ \\
\hline & 80 & $3.63 \pm 0.80^{c}$ & $112.01 \pm 17.68^{\mathrm{b}}$ & $133.65 \pm 32.87^{b}$ \\
\hline & 160 & $3.34 \pm 0.70^{\mathrm{c}}$ & $120.29 \pm 22.16^{c}$ & $163.28 \pm 35.18^{c}$ \\
\hline
\end{tabular}

${ }^{\mathrm{a}} \mathrm{P}<0.01$ compared with normal group; ${ }^{\mathrm{b}} \mathrm{P}<0.05,{ }^{\mathrm{c}} \mathrm{P}<0.01$ compared with model group.

A
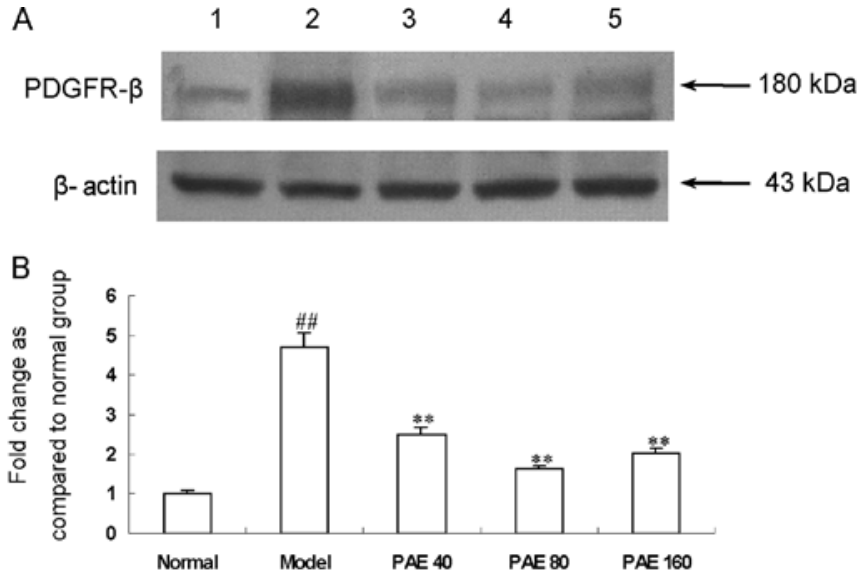

Figure 4. Effects of PAE on the expression of PDGFR- $\beta$ in liver fibrotic rats induced by porcine serum. (A) Representative Western blot image and (B) bar graph of protein quantification plotted from not $<3$ independent experiments. The band intensity of PDGFR- $\beta$ was quantified by densitometry and normalized to $\beta$-actin. Densitometry values in the histograms were expressed as fold change relative to the control (lane 1), which was assigned a value of 1. Lane 1, normal rats; lane 2, immunological hepatic fibrosis rats; lanes 3-5, immunological hepatic fibrosis rats treated with PAE 40, 80, $160 \mathrm{mg} \cdot \mathrm{kg}^{-1}$ respectively. ${ }^{\# \#} \mathrm{P}<0.01$ compared with lane $1 ;{ }^{* *} \mathrm{P}<0.01$ compared with lane 2 .

PDGFR- $\beta$ in the experimental liver were examined because its critical role in the development of liver fibrosis (20). The protein expression of PDGFR- $\beta$ was elevated in the liver of
PS-injected rats (Fig. 4). Administration of PAE significantly reduced the protein level of PDGFR- $\beta$.

Effect of PAE on the activation of MAPK in PS-injected rats. To determine whether the MAPK pathway is modulated during the PS-induced liver fibrosis and the effects of PAE on the activation of MAPK, next we examined three MAPK family members, ERK1/2, p38 and JNK in the rat liver using Western blot analysis. p-ERK1/2, p-p38 and p-JNK showed significantly elevated levels after PS injection, while no changes in ERK1/2, p38 and JNK levels were detected (Fig. 5). Comparing the ratios of p-ERK1/2:ERK1/2, p-p38:p38 and p-JNK:JNK revealed that p-ERK1/2, p-p38 and p-JNK levels decreased significantly during PAE treatment, indicating that ERK1/2, p38 and JNK activation was attenuated by PAE administration.

\section{Discussion}

Hepatic fibrosis is a dynamic response to chronic liver injury by various agents, such as viruses (including hepatitis B and hepatitis C), alcohol, and metabolic and autoimmune disorders. It is well known that constant fibrosis can lead to the development of hepatocellular carcinoma. Interrupting and/or reversing hepatic fibrosis may be a new approach for improving its progression to hepatocellular carcinoma (21). At present, there is no effective treatment of liver fibrosis available for clinical use. Some experimental studies have reported partial success with natural 


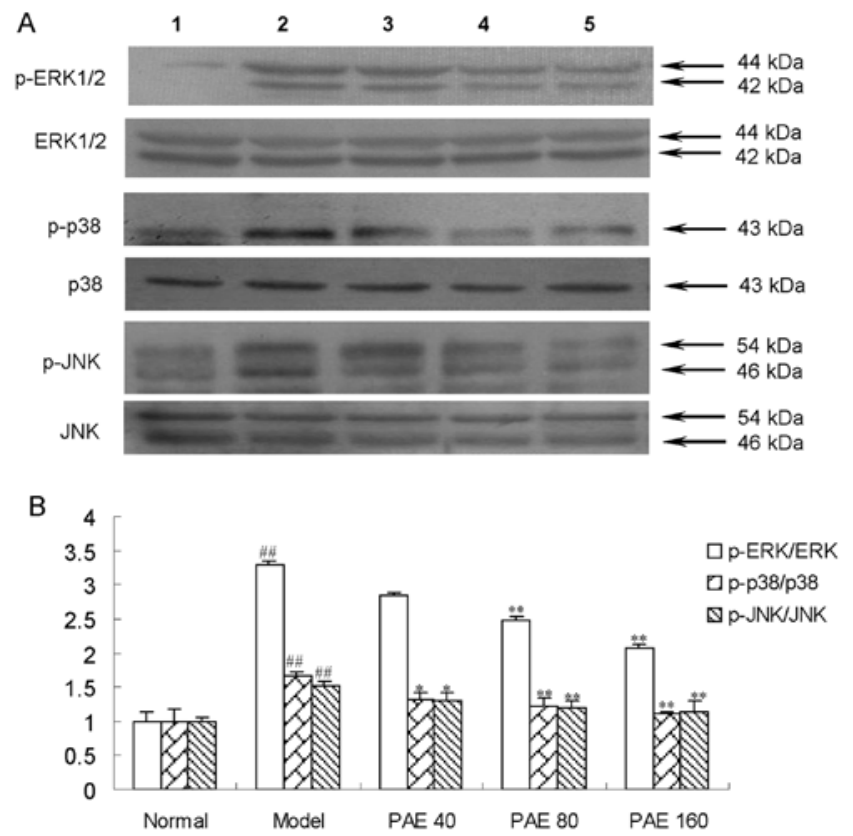

Figure 5. Effect of PAE on ERK1/2, p38 and JNK phosphorylation in liver tissue of immunological hepatic fibrosis rats induced by porcine serum. (A) Representative Western blot image and (B) bar graph of protein quantification plotted from at least 3 independent experiments. The band intensities of phosphorylation of ERK1/2, p38, JNK were normalized to that of the corresponding non-phosphorylated. Densitometry values in the histograms were expressed as fold change relative to the control (lane 1), which was assigned a value of 1 . Lane 1 , normal rats; lane 2, immunological hepatic fibrosis rats; lanes 3-5, immunological hepatic fibrosis rats treated with PAE 40, 80, $160 \mathrm{mg} \cdot \mathrm{kg}^{-1}$ respectively. ${ }^{\# \#} \mathrm{P}<0.01$ compared with lane $1 ;{ }^{*} \mathrm{P}<0.05,{ }^{* *} \mathrm{P}<0.01$ compared with lane 2.

products $(22,23)$. An effective therapeutic approach against the development of hepatic fibrosis is still needed.

Considerable efforts have been made to create appropriate animal models of liver fibrosis for the study of the underlying molecular mechanisms in vivo and to evaluate antifibrotic drugs. Most of the hepatic fibrosis models are so-called post-necrotic hepatic fibrosis, while PS-induced rat hepatic fibrosis model is characterized by accompanying moderate hepatocyte damage (24). Histologically, the changes of PS-induced rat hepatic fibrosis were characterized by mononuclear cell infiltration and fibrotic response in the periportal area, followed by the septum formation connecting portal tract with central veins (25). Changes in PS-induced hepatic fibrosis in rats have some similarities to those observed in hepatic diseases in human. Thus, the PS induced model has been used for testing anti-fibrosis drugs, for investigation into pathogenesis of hepatic fibrosis and hepatic physiopathology. The present study demonstrated that PAE had protective effects on liver fibrosis induced by PS in rats. The animal model of PS-induced liver fibrosis was first established. The histological results showed that the normal structure of lobules was destroyed and pseudolobules formed. Moreover, the increased hydroxyproline content in liver and serum HA, PCIII also confirmed the hepatic fibrogenesis in rats. Treatment with PAE effectively decreased these biomarkers. The hepato-protective effect of PAE was further concluded by marked improvement in the histopathological examinations. PAE administration not only reduced collagen deposition, but also ameliorated oxidative stress. In vitro experiments, PAE significantly inhibited HSCs proliferation. Furthermore, PAE administration obviously inhibited the expression of PDGFR- $\beta$ and phosphorylated ERK1/2, p38, JNK. Results from these experiments clearly showed that PAE inhibited the progression of liver fibrosis induced by PS in rats.

Increasing experimental evidence suggests that reactive oxidative species (ROS) play a critical role in the activation of HSCs during liver fibrogenesis and oxidative stress is a key mechanism in chronic liver damage and fibrosis (26). Increased ROS and resulting oxidative stress are commonly detected in livers from patients with alcohol abuse, hepatitis $\mathrm{C}$ virus infection, iron overload, or chronic cholestasis, as well as in most types of experimental liver fibrogenesis (27). Oxidative stress, in particular, lipid peroxidation is an indicator of tissue damage and accelerates collagen synthesis by stimulating HSCs. It also acts as a signaling mediator for transforming growth factor (TGF)- $\beta$, and plays a major role in hepatic fibrosis (28). Fibrosis caused by lipid peroxidation and its products decreased after the administration of antioxidants in animal models (29). Consequently, antioxidants, particularly those of plant origin, have emerged as potent antifibrotic agents. Our previous studies showed that PAE has protective effect on BCG plus LPS induced liver injury in mice through inhibiting oxidative stress (13). To confirm the effect of PAE on oxidative stress in liver fibrosis, we examined the oxidative stress parameters, including SOD, GSH-Px and MDA. Consistent with the previous studies (28), increased liver MDA levels and depressed SOD and GSH-Px activities were observed in the PS-treated rats. The increase in MDA level in liver suggests enhanced lipid peroxidation leading to tissue damage and failure of antioxidant defense mechanisms to prevent the formation of excessive free radicals (30). Administration of PAE could significantly elevate the activity of SOD and GSH-Px, two antioxidant enzymes, and markedly decreased MDA level, the products of lipid peroxidation, in liver fibrosis rats. The results of the present study indicate that PAE might inhibit lipid peroxidation and effectively recover the anti-oxidative defense system in liver fibrosis. It is possible that the mechanism of hepato-protective effects of PAE may be due to its antioxidant action. Furthermore, oxidative stress, particularly lipid peroxidation induces collagen synthesis to aggravate liver fibrosis (31). Hence, the reduction of ECM in PAE-treated group, as evidenced by HA, PCIII and hydroxyproline, was probably related to its anti-oxidant activity.

It is widely recognized that activated HSCs play a pivotal role in development of liver fibrosis. During HSC activation, two major events occur. First, HSCs increase the synthesis and deposition of ECM proteins. Second, the proliferation rate of HSCs increases. The increased number of HSCs during liver fibrogenesis reflects the activity of several growth factors and their cognate tyrosine kinase receptors (32). In particular, platelet-derived growth factor (PDGF)-BB is the most potent proliferating stimulus for HSCs (19). In vitro experiments, PAE markedly suppressed $\left[{ }^{3} \mathrm{H}\right]$-thymidine incorporation of HSCs in the DNA synthesis study with PDGF-BB stimulation, clearly demonstrated that PAE is a potent inhibiting agent of PDGF-BB induced proliferation in HSC-T6 cells. The effects of PDGF are mediated by dimeric transmembrane receptors composed of $\alpha$ and/or $\beta$-subunits with an intrinsic protein tyrosine kinase activity. PDGF/PDGFR interaction, plays a pivotal role in the development of liver fibrosis (33). Expression 
of PDGF and its receptor subunits has been demonstrated to follow acute and chronic diseased human liver tissue in strict correlation to the extent of its necrotic inflammation and fibrotic damage. Therefore, targeting the PDGF/PDGFR axis is considered to be an effective strategy to inhibit the progress of hepatic fibrosis. Yoshiji et al (34) reported that, imatinib mesylate, a clinically used PDGFR tyrosine kinase inhibitor, markedly attenuated liver fibrosis in rats by inhibiting the PDGF-induced proliferation and migration of activated HSCs. It has been reported that the $\beta$-isoform of the PDGF receptor (PDGFR- $\beta$ ), which binds PDGF-BB, is mainly up-regulated along with $\mathrm{HSCs}$ activation in $\mathrm{CCl}_{4}^{-}$and bile duct ligationinduced liver injuries $(35,36)$. Similar to the $\mathrm{CCl}_{4}$ model, we observed that PDGFR- $\beta$ expression was mainly up-regulated during development of liver fibrosis induced by PS. Moreover, the results of the present study clearly indicate that PAE effectively prevented the development of liver fibrosis and this finding was associated with the inhibition of the expression of PDGFR- $\beta$ and HSCs proliferation.

Increasing experimental evidence suggests the involvement of the MAPK signaling pathway in the progression of hepatic fibrosis. Aberrant expression of the MAPK contributes to the pathogenesis of hepatic fibrosis. Although the exact role of MAPKs in hepatic fibrosis has not been fully elucidated, it is known that at least 15 genes predominantly associated with the MAPK signal transduction pathway are up-regulated in the fibrotic liver (37). Increased expression of MAPKs has been previously reported in the liver of common bile duct ligation rats (38). We observed that the phosphorylation of ERK, JNK and p38 significantly increased in PS-injection rats, suggesting that the MAPK activation also mediated the progression of PS-induced liver fibrosis. Furthermore, MAPKs have been shown in close relationship with the production of ECM and activation, proliferation, apoptosis of HSCs. There is a recent report that in renal tubular epithelial cells, ROS mediate TGF- $\beta$-induced ECM deposition directly through activation of MAPKs and that this effect may be effectively prevented by antioxidants (39). We also examined the influence of PAE in MAPK activation and demonstrated a significant downregulation of phosphorylated ERK, JNK and p38, suggesting that the anti-fibrotic effect of PAE is mainly mediated through suppression of MAPK signaling.

In conclusion, results from the present study demonstrated that PAE was effective in the prevention of PS-induced liver fibrosis in rats. The primary mechanisms of this anti-fibrotic effect could be due to its radical scavenging action, antioxidant activity, decreasing the expression of PDGFR- $\beta$, inhibition of HSC proliferation and MAPK activation. Collectively, these results indicate that $\mathrm{PAE}$ is a potential agent for the prevention of liver fibrosis.

\section{Acknowledgements}

This study was supported by grants from the State High Technology Research and Development Program of China (863 program, no. 2002AA2Z3235), Anhui Provincial Natural Science Foundation (no. 090413108) and Research Fund for the Doctor of Anhui Medical Universtity (XJ200804). The authors acknowledge the help of the staff members of the Institute of Clinical Pharmacology, Anhui Medical University in conducting the study.

\section{References}

1. Friedman SL: Evolving challenges in hepatic fibrosis. Nat Rev Gastroenterol Hepatol 7: 425-436, 2010.

2. Jiao J, Friedman SL and Aloman C: Hepatic fibrosis. Curr Opin Gastroenterol 25: 223-229, 2009

3. Popov Y and Schuppan D: Targeting liver fibrosis: strategies for development and validation of antifibrotic therapies. Hepatology 50: 1294-1306, 2009.

4. Cederbaum AI, Lu Y and Wu D: Role of oxidative stress in alcohol-induced liver injury. Arch Toxicol 83: 519-548, 2009.

5. Aboutwerat A, Pemberton PW and Smith A, et al: Oxidant stress is a significant feature of primary biliary cirrhosis. Biochim Biophys Acta 1637: 142-150, 2003.

6. Sastre J, Serviddio G and Pereda J, et al: Mitochondrial function in liver disease. Front Biosci 12: 1200-1209, 2007.

7. Poli G: Pathogenesis of liver fibrosis: role of oxidative stress. Mol Aspects Med 21: 49-98, 2000.

8. Svegliati BG, D'Ambrosio L and Ferretti G, et al: Fibrogenic effect of oxidative stress on rat hepatic stellate cells. Hepatology 27: 720-726, 1998

9. Wang H, Wei W and Wang NP, et al: Effects of total glucosides of peony on immunological hepatic fibrosis in rats. World $\mathrm{J}$ Gastroenterol 11: 2124-2129, 2005.

10. Liu H, Wei W, Sun WY and Li X: Protective effects of astragaloside IV on porcine-serum-induced hepatic fibrosis in rats and in vitro effects on hepatic stellate cells. J Ethnopharmacol 122: 502-508, 2009

11. Nishiyama N, Wang YL and Saito H: Beneficial effects of $\mathrm{S}-113 \mathrm{~m}$, a novel herbal prescription, on learning impairment model in mice. Biol Pharm Bull 18: 1498-1503, 1995.

12. Shao FR, Wei W, Liu H, Sun WY and Li X: Comparison of protective effect of Shaoqiduogan extracted by different methods on immunological liver injury in mice. J Anhui TCM College 26: 21-24, 2007.

13. Sun WY, Wei W, Gui SY, Wu L and Wang H: Protective effect of extract from Paeonia lactiflora and Astragalus membranaceus against liver injury induced by bacillus Calmette-Guerin and lipopolysaccharide in mice. Basic Clin Pharmacol Toxicol 103: 143-149, 2008.

14. Wu L, Wei W, Gui SY and Sun WY: Effects and mechanisms of shaoqiduogan on mice with chemical liver injury. Zhongguo Zhong Yao Za Zhi 31: 1807-1810, 2006.

15. Okuno M, Akita $\mathrm{K}$ and Moriwaki $\mathrm{H}$, et al: Prevention of rat hepatic fibrosis by the protease inhibitor, camostat mesilate, via reduced generation of active TGF-beta. Gastroenterology 120: 1784-1800, 2001.

16. Mitchell AD and Taylor IE: The spectrophotometric determination of hydroxyproline: an analytical investigation. Analyst 95: 1003-1011, 1970.

17. Li Q, Liu DW, Zhang LM, Zhu B, He YT and Xiao YH: Effects of augmentation of liver regeneration recombinant plasmid on rat hepatic fibrosis. World J Gastroenterol 11: 2438-2443, 2005.

18. Vogel S, Piantedosi R and Frank J, et al: An immortalized rat liver stellate cell line (HSC-T6): a new cell model for the study of retinoid metabolism in vitro. J Lipid Res 41: 882-893, 2000.

19. Pinzani M: PDGF and signal transduction in hepatic stellate cells. Front Biosci 7: d1720-d1726, 2002.

20. Brenner DA: Molecular pathogenesis of liver fibrosis. Trans Am Clin Climatol Assoc 120: 361-368, 2009.

21. Povero D, Busletta C and Novo E, et al: Liver fibrosis: a dynamic and potentially reversible process. Histol Histopathol 25: 1075-1091, 2010.

22. D'Argenio G, Amoruso DC and Mazzone G, et al: Garlic extract prevents $\mathrm{CCl}(4)$-induced liver fibrosis in rats: The role of tissue transglutaminase. Dig Liver Dis 42: 571-577, 2010.

23. Hamza AA: Ameliorative effects of Moringa oleifera Lam seed extract on liver fibrosis in rats. Food Chem Toxicol 48: 345-355, 2010.

24. Baba Y, Saeki K, Onodera T and Doi K: Serological and immunohistochemical studies on porcine-serum-induced hepatic fibrosis in rats. Exp Mol Pathol 79: 229-235, 2005.

25. Gotardo BM, Andrade RG, Oliveira LF and Andrade ZA: Production of septal fibrosis of the liver by means of foreign protein injections into rats. Rev Soc Bras Med Trop 36: 577-580, 2003.

26. Gressner OA, Weiskirchen R and Gressner AM: Biomarkers of liver fibrosis: clinical translation of molecular pathogenesis or based on liver-dependent malfunction tests. Clin Chim Acta 381: 107-113, 2007. 
27. Albano E: Alcohol, oxidative stress and free radical damage Proc Nutr Soc 65: 278-290, 2006.

28. Shimizu I, Ma YR and Mizobuchi Y, et al: Effects of Sho-saiko-to, a Japanese herbal medicine, on hepatic fibrosis in rats. Hepatology 29: 149-160, 1999.

29. Vercelino R, Crespo I and de Souza GF, et al: S-nitroso-Nacetylcysteine attenuates liver fibrosis in cirrhotic rats. J Mol Med 88: 401-411, 2010.

30. Drewa G, Krzyzynska-Malinowska E and Wozniak A, et al: Activity of superoxide dismutase and catalase and the level of lipid peroxidation products reactive with TBA in patients with psoriasis. Med Sci Monit 8: R338-R343, 2002.

31. Lee KS, Buck M, Houglum K and Chojkier M: Activation of hepatic stellate cells by TGF alpha and collagen type I is mediated by oxidative stress through c-myb expression. J Clin Invest 96: 2461-2468, 1995.

32. Atzori L, Poli G and Perra A: Hepatic stellate cell: a star cell in the liver. Int J Biochem Cell Biol 41: 1639-1642, 2009.

33. Friedman SL: Mechanisms of hepatic fibrogenesis. Gastroenterology 134: 1655-1669, 2008.

34. Yoshiji H, Noguchi R and Kuriyama S, et al: Imatinib mesylate (STI-571) attenuates liver fibrosis development in rats. Am J Physiol Gastrointest Liver Physiol 288: G907-G913, 2005.
35. Kinnman N, Francoz C and Barbu V, et al: The myofibroblastic conversion of peribiliary fibrogenic cells distinct from hepatic stellate cells is stimulated by platelet-derived growth factor during liver fibrogenesis. Lab Invest 83: 163-173, 2003.

36. Kinnman $\mathrm{N}$, Goria $\mathrm{O}$ and Wendum $\mathrm{D}$, et al: Hepatic stellate cell proliferation is an early platelet-derived growth factor-mediated cellular event in rat cholestatic liver injury. Lab Invest 81: 1709-1716, 2001.

37. Qiang H, Lin Y and Zhang X, et al: Differential expression genes analyzed by cDNA array in the regulation of rat hepatic fibrogenesis. Liver Int 26: 1126-1137, 2006.

38. Svegliati-Baroni G, Ridolfi $\mathrm{F}$ and Caradonna Z, et al: Regulation of ERK/JNK/p70S6K in two rat models of liver injury and fibrosis. J Hepatol 39: 528-537, 2003.

39. Rhyu DY, Yang Y and Ha H, et al: Role of reactive oxygen species in TGF-betal-induced mitogen-activated protein kinase activation and epithelial-mesenchymal transition in renal tubular epithelial cells. J Am Soc Nephrol 16: 667-675, 2005. 